\title{
Alpha effect and diffusivity in helical turbulence with shear
}

\author{
D. Mitra ${ }^{1}$, P. J. Käpylä ${ }^{2}$, R. Tavakol ${ }^{1}$, and A. Brandenburg ${ }^{3}$ \\ 1 Astronomy unit, School of Mathematical Sciences, Queen Mary University of London, Mile End Road, London E1 4NS, UK \\ e-mail: dhruba.mitra@gmail.com \\ 2 Observatory, Tähtitorninmäki (PO Box 14), 00014 University of Helsinki, Finland \\ 3 NORDITA, Roslagstullsbacken 23, 10691 Stockholm, Sweden
}

Received 10 June 2008 / Accepted 28 November 2008

ABSTRACT

\begin{abstract}
Aims. We study the dependence of turbulent transport coefficients, such as the components of the $\alpha$ tensor $\left(\alpha_{i j}\right)$ and the turbulent magnetic diffusivity tensor $\left(\eta_{i j}\right)$, on shear and magnetic Reynolds number in the presence of helical forcing.

Methods. We use three-dimensional direct numerical simulations with periodic boundary conditions and measure the turbulent transport coefficients using the kinematic test field method. In all cases the magnetic Prandtl number is taken as unity.

Results. We find that with increasing shear the diagonal components of $\alpha_{i j}$ quench, whereas those of $\eta_{i j}$ increase. The antisymmetric parts of both tensors increase with increasing shear. We also propose a simple expression for the turbulent pumping velocity (or $\gamma$ effect). This pumping velocity is proportional to the kinetic helicity of the turbulence and the vorticity of the mean flow. For negative helicity, i.e. for a positive trace of $\alpha_{i j}$, it points in the direction of the mean vorticity, i.e. perpendicular to the plane of the shear flow. Our simulations support this expression for low shear and magnetic Reynolds number. The transport coefficients depend on the wavenumber of the mean flow in a Lorentzian fashion, just as for non-shearing turbulence.
\end{abstract}

Key words. magnetohydrodynamics (MHD) - hydrodynamics - turbulence - magnetic fields

\section{Introduction}

Understanding the origin of non-uniform large-scale magnetic fields in stars, galaxies, and accretion discs continues to pose important challenges. Such fields are commonly thought to be the result of dynamo action that converts the kinetic energy of turbulent motions and large-scale shear into magnetic energy. The usual framework for studying such dynamo actions is mean field electrodynamics (e.g. Krause \& Rädler 1980). Over the years, however, the applicability of this framework has been questioned (Piddington 1981; Vainshtein \& Cattaneo 1992). In particular, an important debate in this connection revolves around the role played by magnetic helicity (Gruzinov \& Diamond 1994). Magnetic helicity is conserved for ideal (non-dissipative) magnetohydrodynamics (MHD) and also in the limit of large magnetic Reynolds numbers. This conservation places severe constraints on the growth of the mean magnetic field and may regulate the quenching of the $\alpha$ effect as the magnetic Reynolds number $\left(\mathrm{Re}_{\mathrm{M}}\right)$ increases (Brandenburg \& Subramanian 2005). Under certain circumstances (no magnetic helicity fluxes), $\alpha$ quenching becomes more severe with $\alpha$ proportional to $\mathrm{Re}_{\mathrm{M}}^{-1}$. It has been suggested that this can be alleviated by expelling magnetic helicity through open boundaries (Blackman \& Field 2000; Kleeorin et al. 2000), possibly mediated by shear (Vishniac \& Cho 2001; Subramanian \& Brandenburg 2004; Brandenburg \& Sandin 2004). Furthermore, shear itself is an important ingredient in MHD dynamos in solar and stellar settings as, for example, in the solar tachocline. Hence, it is important to understand how shear affects the turbulent transport coefficients, in particular the components of the $\alpha_{i j}$ and $\eta_{i j}$ tensors.

Several studies have recently looked at various aspects of this problem (see, e.g., Rogachevskii \& Kleeorin 2003, 2004; Brandenburg 2005; Rüdiger \& Kitchatinov 2006; Rädler \& Stepanov 2006; Leprovost \& Kim 2008;
Brandenburg et al. 2008a). These works employ different tools and make different assumptions and are often applicable to limited regions of the parameter space. As a consequence, care must be taken in comparing these results. For example, using semianalytical tools, which treat the nonlinear Lorentz force feedback perturbatively, Leprovost \& Kim (2008) have found that shear can reduce $\alpha$ in helically forced turbulence. This is analogous to the "rotational quenching" of turbulent transport coefficients with increasing Coriolis or inverse Rossby numbers (Kitchatinov et al. 1994; Pipin et al. 1996). There are also other related cases in which the presence of shear enhances the growth rate of the dynamo. For example; using direct numerical simulations of the MHD equations in the presence of shear and non-helical forcing, Yousef et al. (2008a,b) have found largescale dynamos whose growth rate increases linearly with shear. Such scaling has also been found for $\alpha$-shear dynamos where the $\alpha$ effect is due to stratified convection with shear (Käpylä et al. 2008a). Furthermore, using the kinematic test field method (described below), Brandenburg et al. (2008a) have studied the dynamo coefficients in the presence of shear, but in the absence of helicity, and they find that Gaussian fluctuations of $\alpha$ about zero are strong enough to drive an incoherent $\alpha$-shear dynamo (Vishniac \& Brandenburg 1997; Proctor 2007). The significance of the incoherent $\alpha$ effect has also been stressed by Hughes \& Proctor (2009), although their system may have also had a net $\alpha$ effect. They dismissed this on the grounds that for an imposed uniform magnetic field $\alpha$ is very small. However, this result disagrees with recent calculations of $\alpha$ using the test field method (Käpylä et al. 2008b).

In this paper we use three-dimensional direct numerical simulations of the kinematic test field equations with helical forcing in order to study the dependence of turbulent transport coefficients on shear and magnetic Reynolds number. In Sect. 2 
we give a brief account of our model. Section 3 contains our results and finally we conclude in Sect. 4.

\section{The model}

We use the test field method (Schrinner et al. 2005, 2007) to calculate the turbulent transport coefficients. This method and its modification in the presence of large-scale shear are described in Brandenburg et al. (2008a). Here we just point out the essence of the method and elaborate only on those aspects where our treatment differs from their paper.

In the presence of large-scale shear, the equations of magnetohydrodynamics are treated in the following way. Writing the velocity as the sum $\boldsymbol{U}+\boldsymbol{U}^{S}$, where the large-scale shear velocity $\boldsymbol{U}^{S}=S x \hat{\boldsymbol{y}}$ with a constant shear $S$, and assuming an isothermal equation of state characterised by the sound speed $c_{\mathrm{s}}$, the momentum equation becomes

$\frac{\mathcal{D} \boldsymbol{U}}{\mathcal{D} t}=-\boldsymbol{U} \cdot \boldsymbol{\nabla} \boldsymbol{U}-S U_{x} \hat{\boldsymbol{y}}-c_{\mathrm{s}}^{2} \boldsymbol{\nabla} \ln \rho+\boldsymbol{f}+\boldsymbol{F}_{\text {visc }}$.

Here $\boldsymbol{F}_{\text {visc }}=\rho^{-1} \boldsymbol{\nabla} \cdot(2 \rho v \mathbf{S})$ is the viscous force, $\mathbf{S}_{i j}=\frac{1}{2}\left(U_{i, j}+\right.$ $\left.U_{j, i}\right)-\frac{1}{3} \delta_{i j} \boldsymbol{\nabla} \cdot \boldsymbol{U}$ is the traceless rate of strain tensor (not to be confused with the shear parameter $S$ ), $v$ is the kinematic viscosity, $\rho$ is the fluid density, $c_{\mathrm{s}}$ is the isothermal sound speed and

$\frac{\mathcal{D}}{\mathcal{D} t} \equiv \frac{\partial}{\partial t}+S x \frac{\partial}{\partial y}$.

As our external forcing $\boldsymbol{f}$ we employ helical, white-in-time, random forcing described in Brandenburg (2001). In this paper we consider the purely kinematic problem, so there is no feedback in Eq. (1) due to the Lorentz force. In addition to the momentum equation, we have the continuity equation

$\frac{\mathcal{D} \rho}{\mathcal{D} t}=-\boldsymbol{U} \cdot \boldsymbol{\nabla} \ln \rho-\boldsymbol{\nabla} \cdot \boldsymbol{U}$

and the uncurled induction equation in the Weyl gauge,

$\frac{\mathcal{D} \boldsymbol{A}}{\mathcal{D} t}=-S A_{y} \hat{\boldsymbol{x}}+\boldsymbol{U} \times \boldsymbol{B}-\eta \mu_{0} \boldsymbol{J}$.

Here the magnetic field is $\boldsymbol{B}=\boldsymbol{\nabla} \times \boldsymbol{A}$, the current density is $\boldsymbol{J}=\boldsymbol{\nabla} \times \boldsymbol{B} / \mu_{0}$, and $\mu_{0}$ and $\eta$ are the vacuum permeability and the molecular magnetic diffusivity, respectively. In the mean field approach to MHD one usually decomposes the magnetic field (or magnetic vector potential) and velocity into mean (indicated by an overbar) and fluctuating parts respectively

$\boldsymbol{A}=\overline{\boldsymbol{A}}+\boldsymbol{a}, \quad \boldsymbol{U}=\overline{\boldsymbol{U}}+\boldsymbol{u}$.

The equations satisfied by the mean and fluctuating parts of the magnetic vector potential are given by

$\frac{\mathcal{D} \overline{\boldsymbol{A}}}{\mathcal{D} t}=-S \bar{A}_{y} \hat{\boldsymbol{x}}+\overline{\boldsymbol{U}} \times \overline{\boldsymbol{B}}+\overline{\mathcal{E}}-\eta \mu_{0} \overline{\boldsymbol{J}}$

and

$\frac{\mathcal{D} \boldsymbol{a}}{\mathcal{D} t}=-S a_{y} \hat{\boldsymbol{x}}+\overline{\boldsymbol{U}} \times \boldsymbol{b}+\boldsymbol{u} \times \overline{\boldsymbol{B}}+\boldsymbol{u} \times \boldsymbol{b}-\overline{\mathcal{E}}-\eta \mu_{0} \boldsymbol{j}$,

where $\boldsymbol{j}=\boldsymbol{J}-\overline{\boldsymbol{J}}$ and $\overline{\boldsymbol{E}}=\overline{\boldsymbol{u} \times \boldsymbol{b}}$ is the mean turbulent electromotive force. Specification of this second order quantity in terms of the mean field constitutes a closure problem. A common procedure is to expand $\overline{\mathcal{E}}$ in terms of the mean field $\overline{\boldsymbol{B}}$ and its derivatives,

$\overline{\mathcal{E}}_{i}=\overline{\mathcal{E}}_{0 i}+\alpha_{i j} \bar{B}_{j}+\eta_{i j k} \bar{B}_{j, k}$, where $\alpha_{i j}$ and $\eta_{i j k}$ are the tensorial turbulent transport coefficients, and $\overline{\mathcal{E}}_{0}$ quantifies additional contributions that arise even in the absence of a mean field, owing to small-scale dynamo action, for example. Here and throughout, summation is assumed over repeated indices. In the test field method we take the mean magnetic field to be a given "test field" $\overline{\boldsymbol{B}}$, and calculate these tensors by measuring $\overline{\mathcal{E}}$ by solving Eq. (7) simultaneously with Eqs. (1) and (3), while using Eq. (5) to find $\boldsymbol{u}$. In order to find all the components of the $\alpha_{i j}$ and $\eta_{i j}$ tensors, and not just projections relevant to the actual fields, one has to use an orthogonal set of test fields and solve Eq. (7) for each of them.

In our simulations we employ averages over $x$ and $y$ directions to define our mean fields, which are therefore functions of $z$ and $t$ only. Thus, all other components of $\bar{B}_{j, k}$ except $\bar{B}_{x, z}$ and $\bar{B}_{y, z}$ vanish, and the $\alpha$ and $\eta$ tensors can be written as rank two tensors with $\eta_{i 1}=\eta_{i 23}$ and $\eta_{i 2}=-\eta_{i 13}$ for $i, j=(1,2)$ (Brandenburg et al. 2008a). Now consider, as an example, the following two test fields,

$\bar{B}_{i}^{c 1}=B_{0}(\cos k z, 0,0), \quad \mu_{0} \bar{J}_{i}^{c 1}=k B_{0}(0,-\sin k z, 0)$,

$\bar{B}_{i}^{s 1}=B_{0}(\sin k z, 0,0), \quad \mu_{0} \bar{J}_{i}^{s 1}=k B_{0}(0,+\cos k z, 0)$,

which we use to compute the two corresponding mean electromotive forces $\overline{\mathcal{E}}^{c 1}$ and $\overline{\mathcal{E}}^{s 1}$. The relevant components of the $\alpha$ and $\eta$ tensors are then given by

$\left(\begin{array}{c}\alpha_{i 1} \\ -\eta_{i 2} k\end{array}\right)=\left(\begin{array}{cc}\cos k z & \sin k z \\ -\sin k z & \cos k z\end{array}\right)\left(\begin{array}{c}\overline{\mathcal{E}}_{i}^{c 1} \\ \overline{\mathcal{E}}_{i}^{s 1}\end{array}\right)$.

The rest of the $2+2$ components of the $\alpha$ and $\eta$ tensors can be similarly determined by using the test fields,

$\bar{B}_{i}^{c 2}=B_{0}(0, \cos k z, 0), \quad \bar{B}_{i}^{s 2}=k B_{0}(0, \sin k z, 0)$.

In what follows we denote a test field by $\overline{\boldsymbol{B}}^{p q}$, where $p=c, s$ and $q=1,2$. A particular small-scale magnetic vector potential that develops in response to the $\overline{\boldsymbol{B}}^{p q}$ is denoted by $\boldsymbol{a}^{p q}$ and the corresponding small-scale magnetic field is given by $\boldsymbol{b}^{p q}=\boldsymbol{\nabla} \times$ $\boldsymbol{a}^{p q}$. Note that at large values of $\operatorname{Re}_{\mathrm{M}}$ there will also be smallscale dynamo action that will lead to spurious time-dependencies of $\overline{\mathcal{E}}$. However, since neither the test fields nor $\alpha_{i j}$ or $\eta_{i j}$ depend on time, i.e.

$\overline{\mathcal{E}}_{i}^{p q}(z, t)=\overline{\mathcal{E}}_{0 i}^{p q}(z, t)+\alpha_{i j} \bar{B}_{j}^{p q}(z)+\eta_{i j k} \bar{B}_{j, k}^{p q}(z)$,

such time dependence must be entirely due to $\overline{\mathcal{E}}_{0}^{p q}(z, t)$ and can be eliminated by time averaging.

In the following we shall, to begin with, use $k=k_{1}$, the wavenumber corresponding to the box size, to study the dependence of $\alpha_{i j}$ and $\eta_{i j}$ on shear and magnetic Reynolds number. We shall discuss the dependence of the $\alpha_{i j}$ and $\eta_{i j}$ tensors on $k$ in Sect. 3.3. Note that the usual approach of using uniform applied fields for calculating $\alpha$ (e.g., Courvoisier et al. 2006) corresponds to a special case of the test field method for $k=0$. However, dynamos generate large-scale fields with non-zero $k$, so it is important to relax this restriction. It is then also important to calculate $\eta_{i j}$. The test field method allows the simultaneous calculation of all the components of the $\alpha_{i j}$ and $\eta_{i j}$ tensors for arbitrary values of $k$.

The test field method has recently been criticised by Cattaneo \& Hughes (2008) on the grounds that the test fields are arbitrary predetermined mean fields. They argue that the resulting turbulent transport coefficients will only be approximations 

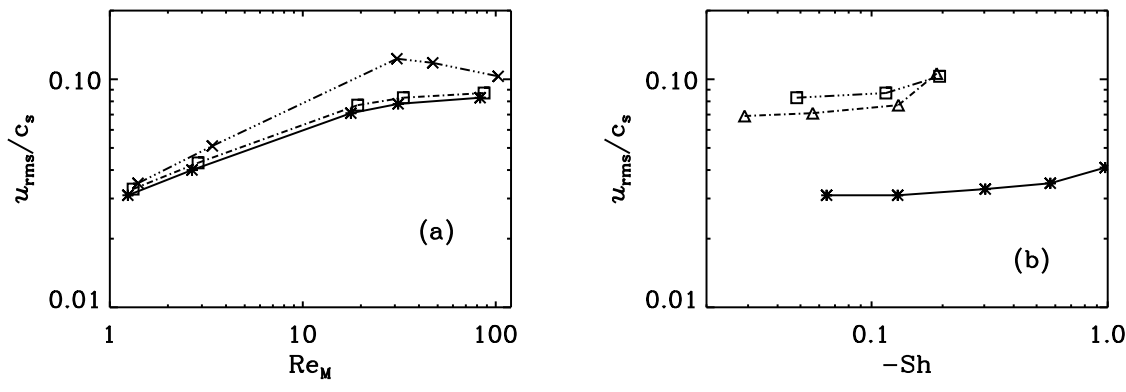

Fig. 1. Root-mean-square velocity $u_{\text {rms }}$ normalized by the speed of sound $c_{\mathrm{s}}$ as: a) a function of $\mathrm{Re}_{\mathrm{M}}$ for different values of Sh: Sh $0.07(*), 0.2(\triangle)$, and $0.3(\square)$ respectively and $\mathbf{b})$ a function of the shear parameter Sh for different value of $\operatorname{Re}_{\mathrm{M}}: \operatorname{Re}_{\mathrm{M}} \sim 1(*), \operatorname{Re}_{\mathrm{M}} \sim 20(\Delta)$, and $\operatorname{Re}_{\mathrm{M}} \sim 72(\square)$. to the true values unless the test fields are close to the actual mean fields - a criticism equally applicable to other methods using arbitrary uniform fields. This concern has been addressed by Tilgner \& Brandenburg (2008), who argue that Eq. (7) can instead be applied to any mean field. This statement has been numerically verified in three cases that we describe below.

Firstly, the test field method correctly reproduces a vanishing growth rate in saturated nonlinear cases (Brandenburg et al. 2008b). Secondly, in the time-dependent case, the test field method correctly reproduces also a non-vanishing growth rate, but in that case it is no longer permissible to express $\overline{\mathcal{E}}$ in terms of a multiplication of turbulent transport coefficients with the mean field and its spatial derivatives. One must therefore write Eq. (8) as a convolution in time (Hubbard \& Brandenburg 2008). Finally, the success of the test field method becomes particularly clear when it is applied to a passive vector field that obeys a separate induction equation with a velocity field from a saturated dynamo (Tilgner \& Brandenburg 2008). This question was originally posed by Cattaneo \& Tobias (2008). In particular for the Roberts flow with a mean field of Beltrami type, e.g. one that is proportional to $\left(\cos k_{1} z, \sin k_{1} z, 0\right)$, the $\alpha_{i j}$ tensor is anisotropic and has an additional component proportional to $\bar{B}_{i} \bar{B}_{j}$ that tends to quench the components of the isotropic part of $\alpha_{i j}$. The fastest growing passive vector field is then proportional to $\left(\sin k_{1} z,-\cos k_{1} z, 0\right)$. This result has been confirmed both numerically and using weakly nonlinear theory (Tilgner \& Brandenburg 2008).

In the following we ignore the complications involving timedependent mean fields and restrict ourselves to transport coefficients that apply strictly speaking only to the time-independent or marginally excited case. For our numerical simulations we use the PENCIL CODE ${ }^{1}$, where the test field algorithm has already been implemented. All our numerical simulations are performed in a periodic cubic box. The forcing scale is chosen to have the wavenumber $k_{\mathrm{f}} / k_{1}=5$. This gives enough scale separation for a large-scale field to develop (Haugen et al. 2004), and is still not too big to reduce the resulting Reynolds numbers too much. We use units such that $c_{\mathrm{s}}=k_{1}=\rho_{0}=\mu_{0}=1$ and arrange the forcing amplitude so that the Mach number is around 0.1. For the magnetic Prandtl number we choose $\operatorname{Pr}_{M}=v / \eta=1$, where $v$ lies in the range $3 \times 10^{-3}$ to $2 \times 10^{-4}$ (in units of $c_{\mathrm{s}} / k_{1}$ ). We choose the shear $S$ such that the parameter

$\mathrm{Sh} \equiv S /\left(u_{\mathrm{rms}} k_{\mathrm{f}}\right)$

takes values in the range -0.02 to -0.9 , where $u_{\mathrm{rms}}$ is the rootmean-square velocity. All the runs are started with uniform density, $\rho=\rho_{0}, \boldsymbol{U}=0$, and $\boldsymbol{a}^{p q}=0$. Depending upon the parameters of a particular run we use up to $256^{3}$ grid points. For each test field $\overline{\boldsymbol{B}}^{p q}$ we calculate the time averages of the $\alpha_{i j}$ and $\eta_{i j}$ tensors

${ }^{1}$ http://www.nordita.org/software/pencil-code over time intervals over which $u_{\mathrm{rms}}$ is statistically stationary. In runs with higher magnetic Reynolds numbers,

$\operatorname{Re}_{\mathrm{M}} \equiv u_{\mathrm{rms}} /\left(\eta k_{\mathrm{f}}\right)$

we obtain an exponential growth of small-scale magnetic field (see below). We interpret this as being associated with the $\overline{\mathcal{E}}_{0}^{p q}(z, t)$ term. This often gives rise to large fluctuations in all components of the $\alpha_{i j}$ and $\eta_{i j}$ tensors at late times. In such cases we confine our calculations of the time averages to time intervals over which $\boldsymbol{b}_{\text {rms }}^{p q}$ does not exceed $\overline{\boldsymbol{B}}^{p q}$ by more than a factor of about 20. Up until this point, the components of the $\alpha_{i j}$ and $\eta_{i j}$ tensors show only their intrinsic fluctuations, but at later times these will be swamped by additional contributions from the small-scale dynamo that grows exponentially in time. If necessary, we repeat our calculations over several independent realizations by resetting $\boldsymbol{a}^{p q}=0$ at regular time intervals.

\section{Results}

Our principal aim in this paper is to study the effects of varying shear (Sh) and magnetic Reynolds number $\left(\mathrm{Re}_{\mathrm{M}}\right)$ on the components of the $\alpha_{i j}$ and $\eta_{i j}$ tensors. In the subsection below we summarize our results concerning the different components of these tensors.

\subsection{Diagonal components of the transport tensors}

The isotropic parts of the $\alpha_{i j}$ and $\eta_{i j}$ tensors are respectively characterised as

$\alpha=\frac{1}{2}\left\langle\alpha_{11}+\alpha_{22}\right\rangle, \quad \eta_{\mathrm{t}}=\frac{1}{2}\left\langle\eta_{11}+\eta_{22}\right\rangle$,

where $\langle\cdot\rangle$ denotes an average taken over $z$ and $t$. We normalize these quantities by $\alpha_{0}=-\frac{1}{3} u_{\mathrm{rms}}$ and $\eta_{\mathrm{t} 0}=\frac{1}{3} u_{\mathrm{rms}} / k_{\mathrm{f}}$ which are their respective expressions obtained using the First Order Smoothing Approximation (FOSA) for $\mathrm{Re}_{\mathrm{M}} \ll 1$, which has previously been confirmed with the test field method in simulations of helical turbulence without shear (Sur et al. 2008).

Since $u_{\mathrm{rms}}$ enters the normalization of $\alpha_{i j}$ and $\eta_{i j}$, it is useful to first look at how it changes as a function of $\operatorname{Re}_{M}$ (Fig. 1a) and Sh (Fig. 1b). As can be seen, $u_{\mathrm{rms}}$ increases as a function of $\mathrm{Re}_{\mathrm{M}}$ for small $\operatorname{Re}_{M} \sim 1$ and then reaches a plateau for high $\mathrm{Re}_{\mathrm{M}}$. On the other hand, $u_{\mathrm{rms}}$ is almost a constant as a function of shear except for high $\mathrm{Sh}$, where we observe excitation of the vorticity dynamo discussed further in Sect. 3.4.

In Fig. 2 we show $\alpha$ as a function of shear for three different values of the magnetic Reynolds number $\operatorname{Re}_{\mathrm{M}} \approx 1,20$ and 72 . Figure $2 \mathrm{~b}$ shows the corresponding results for $\eta_{\mathrm{t}}$. We note that for small shear the turbulent transport coefficients are close to their values for zero shear. As $|\mathrm{Sh}|$ increases, $\alpha$ decreases and $\eta_{\mathrm{t}}$ increases up to four times $\eta_{\mathrm{t} 0}$. As can be seen there is a clear 

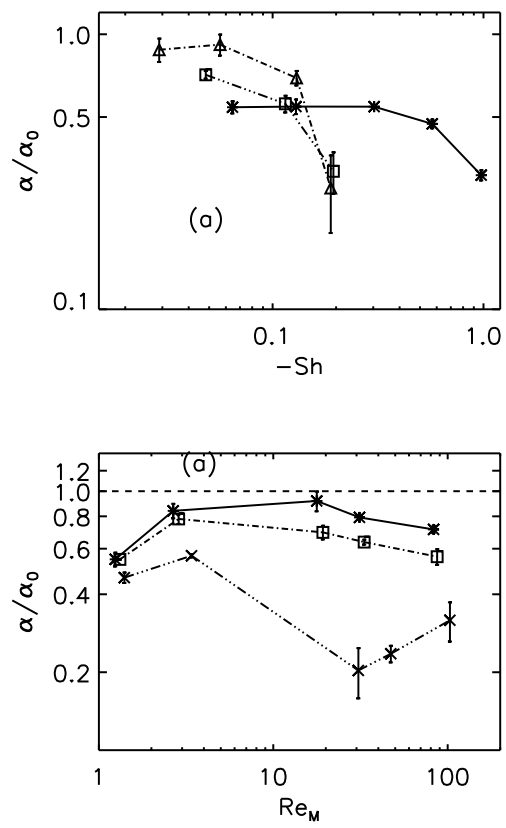
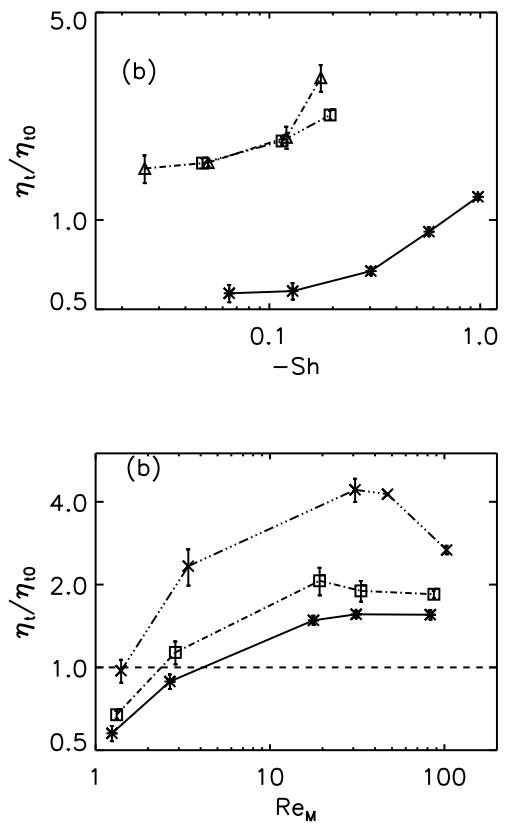

Fig. 2. Turbulent transport coefficients a) $\alpha / \alpha_{0}$ and b) $\eta_{\mathrm{t}} / \eta_{\mathrm{t} 0}$ as functions of shear parameter $\mathrm{Sh}$ for different values of $\operatorname{Re}_{\mathrm{M}}: \operatorname{Re}_{\mathrm{M}} \sim 1(*), \operatorname{Re}_{\mathrm{M}} \sim$ $20(\triangle)$, and $\operatorname{Re}_{\mathrm{M}} \sim 72(\square)$.

Fig. 3. a) $\alpha / \alpha_{0}$ and b) $\eta_{\mathrm{t}} / \eta_{\mathrm{t} 0}$ as functions of $\mathrm{Re}_{\mathrm{M}}$, for different values of $\mathrm{Sh}$ : Sh $\sim 0.07(*), 0.2(\triangle)$, and $0.3(\square)$ respectively. Horizontal dashed lines at $\alpha / \alpha_{0}=1$ and $\eta_{\mathrm{t}} / \eta_{\mathrm{t} 0}=1$ are added to facilitate comparison. reduction (quenching) of $\alpha$ with increasing shear in all these cases. In order to examine the possible convergence of the results with $\operatorname{Re}_{\mathrm{M}}$ we plot $\alpha$ and $\eta_{\mathrm{t}}$ as functions of $\mathrm{Re}_{\mathrm{M}}$ for three different values of the shear parameter Sh, see Fig. 3. For small $\operatorname{Re}_{\mathrm{M}} \sim 1$ we observe an increase in both $\alpha$ and $\eta_{\mathrm{t}}$ with $\operatorname{Re}_{\mathrm{M}}$. A similar initial increase of $\alpha$ and $\eta_{\mathrm{t}}$ was also seen in earlier simulations of helical turbulence without shear (Sur et al. 2008) and in non-helical shear flow turbulence (Brandenburg et al. 2008a). For higher values of $\mathrm{Re}_{\mathrm{M}}$ and Sh, both $\alpha$ and $\eta_{\mathrm{t}}$ show large variations. In these kinematic simulations, however, we expect them to tend to constant values asymptotically at high $\operatorname{Re}_{\mathrm{M}}$ (Sur et al. 2008).

In an earlier study of shear flow turbulence with non-helical forcing by Brandenburg et al. (2008a), the diagonal components of $\eta_{i j}$ were found to be the same. Obviously, in the absence of helicity all the components of $\alpha_{i j}$ are zero. It turns out that in the presence of helicity the two diagonal components of $\eta_{i j}$ are still the same, but those of $\alpha_{i j}$ are now non-zero and not equal to each other. This is best shown by considering the quantities

$\epsilon_{\alpha}=\frac{1}{2}\left\langle\alpha_{11}-\alpha_{22}\right\rangle, \quad \epsilon_{\eta}=\frac{1}{2}\left\langle\eta_{11}-\eta_{22}\right\rangle$.

The results are shown in Fig. 4. Note that especially for large values of $\operatorname{Re}_{\mathrm{M}}$ the values of $\epsilon_{\alpha}$ are predominantly negative. Since both $\alpha_{11}$ and $\alpha_{22}$ are negative, this means that $\left|\alpha_{11}\right|$ is larger than $\left|\alpha_{22}\right|$, although the relative difference is only of the order of at most 5 per cent.

\subsection{Off-diagonal components}

We now consider the off-diagonal components of $\alpha_{i j}$ and $\eta_{i j}$. The results are depicted in Figs. 5 and 6. Of particular interest among these is the component $\eta_{21}$, whose dependence on $\mathrm{Sh}$ and $\mathrm{Re}_{\mathrm{M}}$ is shown in Figs. $5 \mathrm{~d}$ and $6 \mathrm{~d}$, respectively. This component can indicate the possible presence of a shear-current dynamo that may operate when $\eta_{21} S /\left(\eta_{\mathrm{T}} k_{1}\right)^{2}>1$ (Rogachevskii \& Kleeorin 2003, 2004). Here $\eta_{\mathrm{T}}=\eta_{\mathrm{t}}+\eta$ is the total (sum of turbulent and microscopic) magnetic diffusivity. In our case we have $S<0$, which implies that the necessary condition for the shear-current dynamo to operate is $\eta_{21}<0$. As can be seen from Fig. 6d, for the range of $\operatorname{Re}_{M}$ values considered here $\eta_{21}$ is positive for small shear but becomes negative for strong shear and certain values of $\mathrm{Re}_{\mathrm{M}}$. Earlier work of Brandenburg et al. (2008a) without helicity did indicate a similar sign change, although only for larger $\mathrm{Re}_{\mathrm{M}}$. However, the error bars were so large that this result could not be regarded as significant. For the run with the strongest shear $(-\mathrm{Sh} \approx 0.3)$ and for $\mathrm{Re}_{\mathrm{M}}=40$ we now find $\eta_{21}$ to be more clearly negative, but for smaller and larger values of $\mathrm{Re}_{\mathrm{M}}$ the results are again, at least within error bars, compatible with zero. Also, of course, the present results apply to the case with helicity and are therefore not really comparable with those of Brandenburg et al. (2008a), where the helicity is zero. The antisymmetric contributions to the $\alpha_{i j}$ and $\eta_{i j}$ tensors are characterised by the vectors

$\gamma_{k}=-\frac{1}{2} \epsilon_{i j k} \alpha_{i j}, \quad \delta_{k}=-\frac{1}{2} \epsilon_{i j k} \eta_{i j}$

Since our averages depend only on $z$, the $z$ components of these tensors are irrelevant and therefore only the $z$ components of the $\gamma$ and $\delta$ vectors are of interest. We denote those simply by $\gamma$ and $\delta$, with

$\gamma=\frac{1}{2}\left\langle\alpha_{21}-\alpha_{12}\right\rangle, \quad \delta=\frac{1}{2}\left\langle\eta_{21}-\eta_{12}\right\rangle$

A time series of both quantities is shown in Fig. 7 for positive and negative signs of the kinetic helicity. The results show that, in our case with $S<0, \gamma$ is positive (negative) for positive (negative) kinetic helicity, whilst $\delta$ has always the same sign.

Some idea about the functional forms of $\gamma$ and $\delta$ can be obtained from symmetry considerations. The vectors $\gamma$ and $\delta$ enter the electromotive force thus

$\overline{\mathcal{E}}=\ldots+\gamma \times \overline{\boldsymbol{B}}-\mu_{0} \boldsymbol{\delta} \times \overline{\boldsymbol{J}}$,

so we see that $\gamma$ must be a polar vector and $\delta$ must be an axial vector. Using the shear flow $\overline{\boldsymbol{U}}^{S}$, the only axial vector that can be constructed is the mean vorticity, $\overline{\boldsymbol{W}}=\boldsymbol{\nabla} \times \overline{\boldsymbol{U}}^{S}$, so we expect $\boldsymbol{\delta}$ to have a component that is proportional to $\bar{W}$. Likewise, since $\gamma$ is a polar vector which points in the direction of $\bar{W}$, the two must be related via a pseudoscalar. In the present case the only pseudoscalar available is the kinetic helicity, $\overline{\boldsymbol{\omega} \cdot \boldsymbol{u}}$. Based on these symmetry arguments we write

$\gamma=C_{\gamma} \tau^{2} \overline{\omega \cdot u} \bar{W}, \quad \delta=C_{\delta} \tau^{2} \overline{u^{2}} \bar{W}$ 

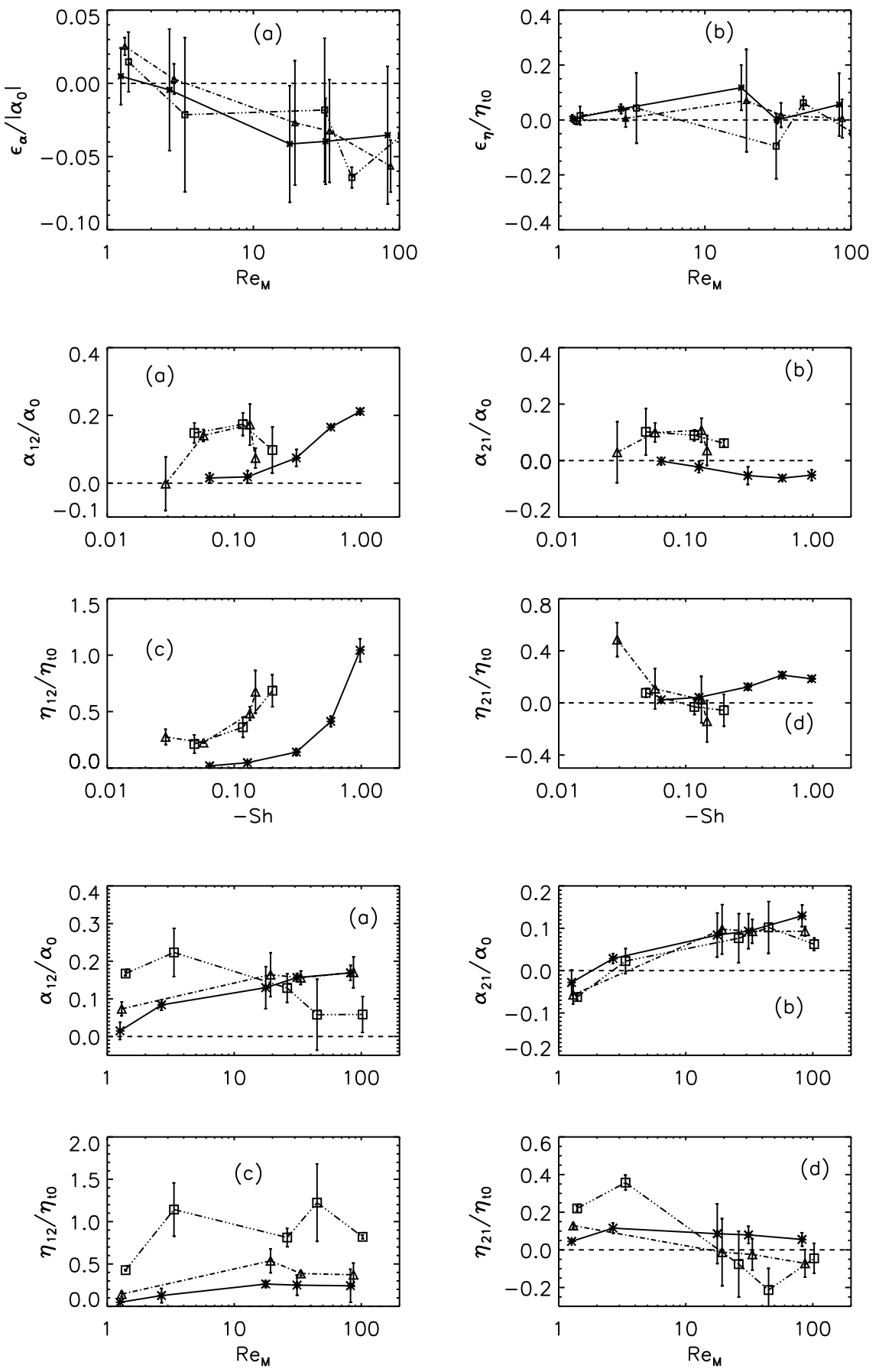
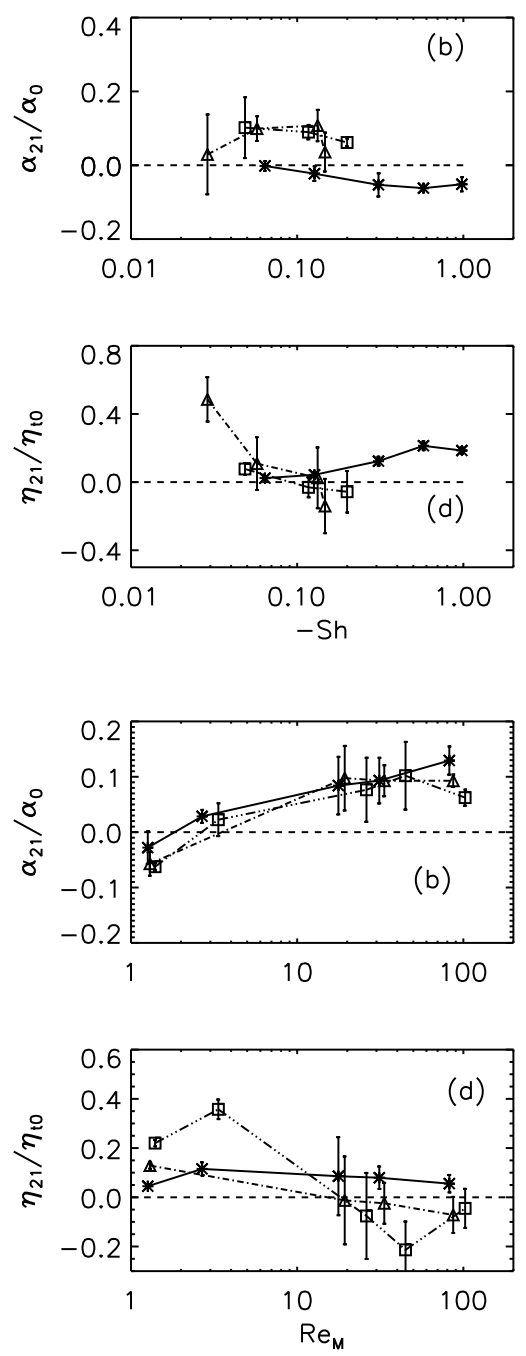

Fig. 4. Normalized a) $\epsilon_{\alpha}$ and b) $\epsilon_{\eta}$ ), as defined in Eq. (17), as functions of $\mathrm{Re}_{\mathrm{M}}$ for constant value of the shear parameter Sh: $\mathrm{Sh} \sim 0.07(*), 0.2(\triangle)$, and $0.3(\square)$ respectively. Horizontal dashed lines are added to facilitate comparison.

Fig. 5. Normalized off-diagonal components of the $\alpha$ and $\eta$ tensors as functions of the shear parameter $S h$ for different values of $\operatorname{Re}_{\mathrm{M}}$ : $\operatorname{Re}_{\mathrm{M}} \sim$ $1(*), \operatorname{Re}_{\mathrm{M}} \sim 20(\triangle)$, and $\operatorname{Re}_{\mathrm{M}} \sim 72(\square)$. Horizontal dashed lines are added to facilitate comparison.

Fig. 6. Normalized off-diagonal components of the $\alpha$ and $\eta$ tensors as functions of $\mathrm{Re}_{\mathrm{M}}$ for constant values of Sh: Sh $0.07(*), \quad 0.2(\triangle)$, and $0.3(\square)$. Horizontal dashed lines are added to facilitate comparison. where we have introduced two non-dimensional quantities, $C_{\gamma}$ and $C_{\delta}$ which could be either positive or negative, and $\tau$ is a correlation time that we approximate here by

$\tau=\left(u_{\mathrm{rms}} k_{\mathrm{f}}\right)^{-1}$.

In the present case, $\overline{\boldsymbol{\omega} \cdot \boldsymbol{u}}$ itself is a negative multiple of $\alpha$. We therefore expect $\gamma$ to have a component proportional to $\alpha \overline{\boldsymbol{W}}$, multiplied by the correlation time $\tau$. By similar arguments we expect $\delta$ to have a component proportional to $\eta_{\mathrm{t}} \overline{\boldsymbol{W}}$, multiplied by $\tau$. Based on these arguments we can also write

$\boldsymbol{\gamma}=\tilde{C}_{\gamma} \alpha \overline{\boldsymbol{W}} \tau, \quad \delta=\tilde{C}_{\delta} \eta_{\mathrm{t}} \overline{\boldsymbol{W}} \tau$,

with new non-dimensional quantities, $\tilde{C}_{\gamma} \approx C_{\gamma} / 3$ and $\tilde{C}_{\delta} \approx$ $C_{\delta} / 3$, that we expect to be of order unity. Our simulations confirm this reasoning and show that both coefficients are of order unity with positive $\tilde{C}_{\gamma}$ and negative $\tilde{C}_{\delta}$ (e.g., $\tilde{C}_{\gamma}=0.5$ and $\tilde{C}_{\delta}=0.25$ for the run shown in Fig. 8) for runs with small $\operatorname{Re}_{\mathrm{M}}$ and Sh; see Figs. 8 and 9, respectively. The sign in Eq. (23) has been chosen a posteriori from our simulations. For high shear and Reynolds number this simple reasoning is no longer accurate and, at least in one case (Fig. 9a), $C_{\gamma}$ even becomes negative. For lower values of Sh we find an almost constant negative $\gamma$ of the order of $0.05 \alpha_{0}$ (or less). This result is fairly independent of $\operatorname{Re}_{\mathrm{M}}$. The moduli of $\gamma$ and $\delta$ increase with increasing shear parameter, and for larger values of $\mathrm{Re}_{\mathrm{M}}$ both quantities may approach an asymptotic value. For small $\mathrm{Re}_{\mathrm{M}}$ we observe a roughly linear increase of $\gamma$ with shear (see inset of Fig 8a), further verifying Eq. (21). 

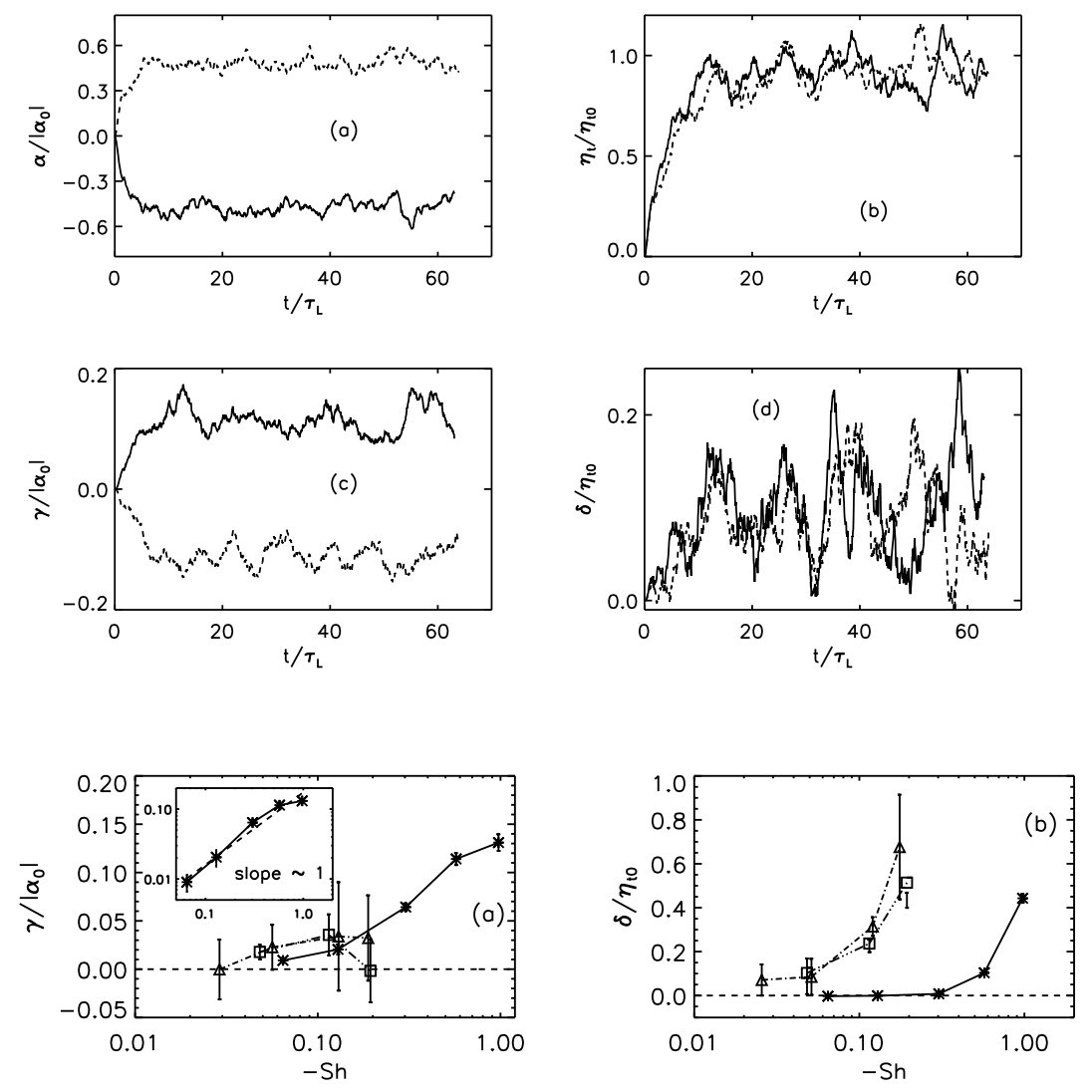

Fig. 7. Normalized time series of turbulent transport coefficients, a) $\alpha /\left|\alpha_{0}\right|$, b) $\eta_{t} / \eta_{t 0}$, c) $\gamma /\left|\alpha_{0}\right|$ and d) $\delta / \eta_{t 0}$, from two runs with exactly the same parameters $\left(\mathrm{Re}_{\mathrm{M}} \sim 1.4, \mathrm{Sh}=-0.5\right)$, but different signs of helicity for the external force. Continuous and broken lines denote results from runs with positive and negative helicities, respectively.

Fig. 8. Normalized a) $\gamma$ and b) $\delta$ as functions of Sh for $\operatorname{Re}_{\mathrm{M}} \approx 1(*), \mathrm{Re}_{\mathrm{M}} \approx 20(\triangle)$, and $\operatorname{Re}_{\mathrm{M}} \approx 72(\square)$. Horizontal dashed lines are added to facilitate comparison. The inset shows $\gamma /\left|\alpha_{0}\right|$ versus $-\mathrm{Sh}$ for $\mathrm{Re}_{\mathrm{M}} \sim 1$.
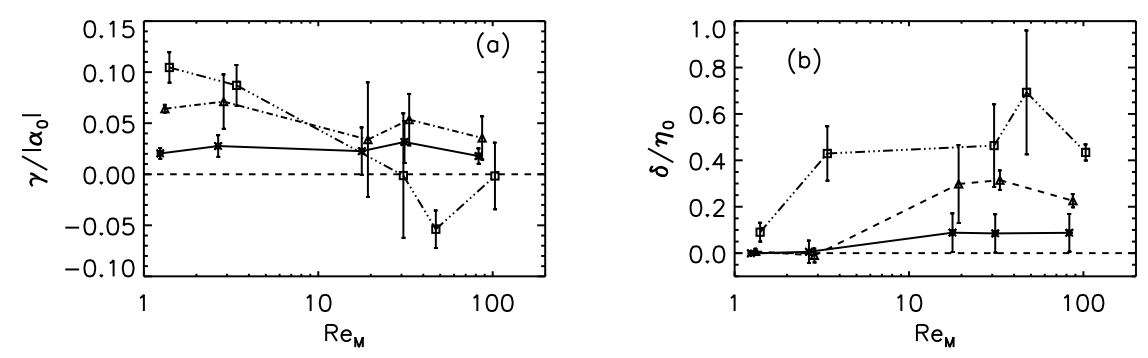

Fig. 9. Normalized a) $\gamma$ and b) $\delta$ as functions of $\operatorname{Re}_{\mathrm{M}}$ for constant values of the shear parameter Sh: Sh $~ 0.07(*), 0.2(\triangle)$, and $0.3(\square)$. Horizontal dashed lines are added to facilitate comparison.

The coefficient $\gamma$ can be interpreted as turbulent pumping, i.e. advection of the magnetic field by means other than the mean velocity field. In strongly stratified convection, turbulent pumping has been seen to be directed from higher to lower turbulence intensity (Tobias et al. 1998, 2001; Ossendrijver et al. 2002; Käpylä et al. 2006), which is usually in the downward direction. Thus, turbulent pumping is likely to play an important role in convection zones of stars where it can overcome the buoyancy of the magnetic field. In the present case where the turbulence is homogeneous, however, stratification does not play a role and the pumping is just due to the combined action of shear and helical turbulence.

\subsection{Scale-dependence}

So far we have confined the calculations of the $\alpha_{i j}$ and $\eta_{i j}$ tensors to test fields whose characteristic length scale is the largest scale in the domain, i.e., test fields of the form $\sin k z$ or $\cos k z$ with $k=k_{1}$, where $k_{1}=2 \pi / L$ and $L$ is the box size of our simulations. We have also done similar calculations for other values of $k$, which correspond to test fields with different characteristic length scales. In Fig. 10 we show the dependence of $\alpha$ and $\eta_{t}$ on $k$ for $\mathrm{Re}_{\mathrm{M}} \approx 3$. The decrease can be modelled by a Lorentzian peaked at $k=0$ (Fig. 10). This result is not surprising, because Lorentzian fits have been obtained earlier also in the absence of shear (Brandenburg et al. 2008c).

\subsection{Effects from the vorticity dynamo}

In the runs with relatively high values of shear and Reynolds number, the root-mean-square velocity, $U_{\text {rms }}$, which initially reaches a steady state, shows an exponential growth at late times, as can be seen in Fig. 11a. Similar behaviour is seen for turbulent transport coefficients, which also show large fluctuations for later times. Large fluctuations of the turbulent transport coefficients at later times are also seen by Sur et al. (2008), but those fluctuations are more irregular and have a different origin. They are interpreted as being due to the development of a small-scale dynamo at late times and are not due to the more systematic increase in the rms velocity, which in turn is 

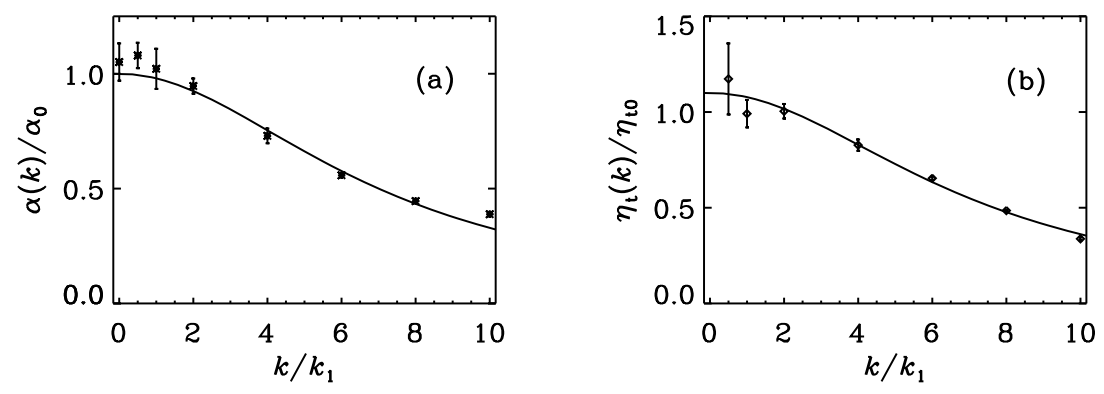

Fig. 10. a) $\alpha(k) / \alpha_{0}$ and b) $\eta_{t}(k) / \eta_{t 0}$ as a function of the wavenumber $k$ of the large-scale magnetic field for $\mathrm{Re}_{\mathrm{M}} \approx 3$. The solid lines show the Lorentzian fits of width $1.4 k_{\mathrm{f}}$.

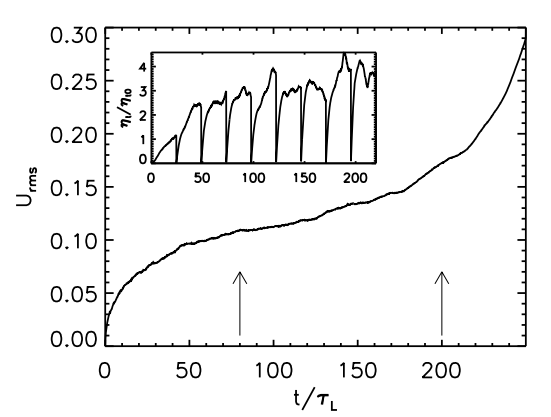

(b)

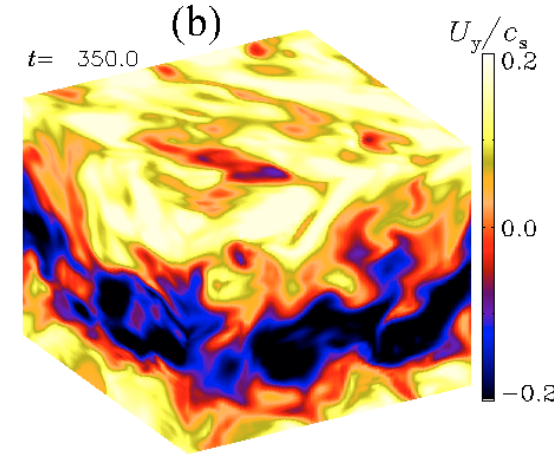

Fig. 11. Panel a) shows the root-mean-square values of the velocity for one of our runs (with $\mathrm{Re} \approx 45$ and $\mathrm{Sh} \approx-0.3$ ) which exhibits a vorticity dynamo. The inset shows the time-series for $\eta_{\mathrm{t}}$. Panel b) shows a pronounced pattern in $U_{y}$ on the periphery of the box at the end of the same run. Similar structures are also found in the $x$ component of the velocity. associated with the shear. However, we emphasize that even before $\boldsymbol{b}^{p q}$ becomes dominated by this type of dynamo action, the temporal and spatial fluctuations of $\alpha_{i j}$ and $\eta_{i j}$ are of the order of $\alpha_{0}$ and $\eta_{\mathrm{t} 0}$, respectively. This is true even for large values of $\mathrm{Re}_{\mathrm{M}}$; see Brandenburg et al. (2008a), who have argued that these fluctuations also contribute to large-scale dynamo action via the incoherent $\alpha$ effect (Vishniac \& Brandenburg 1997; Proctor 2007). For example, the time-series of $\eta_{\mathrm{t}}$ shown in the inset of Fig. 11 has a plateau even beyond the range over which $U_{\text {rms }}$ is steady.

The vertical spikes in the inset of Fig. 11 come from resetting $\boldsymbol{a}^{p q}$ to zero in regular time intervals; see the end of Sect. 2. Note, however, that the mean values and also the upper envelope trace the evolution of $U_{\text {rms }}$ reasonably well, including the increased rise after $t=300$. The late time behaviour is accompanied by the formation of large-scale vortical structures, as seen in Fig. 11b, which is a signature of the vorticity dynamo proposed by Elperin et al. (2003), see also Yousef et al. (2008a,b). A detailed numerical study of the vorticity dynamo has been performed by Käpylä et al. (2008).

The presence of the vorticity dynamo and the resulting systematic variation in $U_{\mathrm{rms}}$ as well as the turbulent transport coefficients for late times limit the lengths of time over which average values of the components of the $\alpha_{i j}$ and $\eta_{i j}$ tensors can be calculated. In the above case this interval lies between the two arrows in Fig. 11a. This limits the range of $\mathrm{Sh}$ and $\mathrm{Re}_{\mathrm{M}}$ that we have probed and explains the larger error bars shown for example in Fig. 6, and why they cannot be reduced by simply running our simulations for longer times. This problem would be avoided in the presence of magnetic fields, because then the resulting Lorentz force would quench the vorticity dynamo (Käpylä \& Brandenburg 2008). This is however beyond the scope of this paper.

\section{Conclusions}

We have studied the effects of varying shear and magnetic Reynolds number on the turbulent transport coefficients in the presence of helicity in the kinematic limit. We have shown that for fixed $\mathrm{Re}_{\mathrm{M}}, \alpha$ is reduced (quenched) with increasing shear. Despite the differences in the assumptions made, this quenching is qualitatively similar to the recent results obtained by Leprovost \& Kim (2008). To the best of our knowledge this is the first numerical study to show quenching of $\alpha$ as a function of shear in helical turbulence. We find that $\eta_{\mathrm{t}}$ increases with increasing shear in the range of $\mathrm{Re}_{M}$ values that we have considered here. A similar behaviour for $\eta_{\mathrm{t}}$ was also seen in Brandenburg et al. (2008a) where the forcing was non-helical.

We also compute the off-diagonal components of the $\alpha_{i j}$ and $\eta_{i j}$ tensors. The antisymmetric part of $\alpha_{i j}$ corresponds to a turbulent pumping velocity $\gamma$ in the direction perpendicular to the plane of the shear flow. It shows a roughly linear increase with shear for small magnetic Reynolds number. We propose simple expressions for $\gamma$ and $\delta$ in Eq. (21) which show reasonable agreement with our numerical results for small Sh and $\mathrm{Re}_{\mathrm{M}}$. Our expression shows that for negative helicity, $\gamma$ points in the direction of the vorticity of the mean flow. Regarding the component $\eta_{21}$ we find indications that, at least in one or two cases, this component changes sign and becomes negative. This could be of significance in connection with the shear-current effect.

We also find that all the turbulent transport coefficients depend on the wavenumber of the mean flow in a Lorentzian fashion, just as in the case of non-shearing turbulence (Brandenburg et al. 2008c). This means that the kinematic values of $\alpha$ and $\eta_{\mathrm{t}}$ for $k=k_{1}$ are close to the values obtained for $k \rightarrow$ 0 . This is not the case for certain non-turbulent flows such as the Galloway-Proctor flow (Courvoisier 2008; Rädler \& Brandenburg 2009). In an earlier paper, Courvoisier et al. (2006) considered only the limiting case $k=0$ for this flow.

Several aspects of the present investigations could be of astrophysical relevance. Turbulence in celestial bodies is helical and exhibits an $\alpha$ effect. In addition, shear $(S)$ can be an important ingredient in that the efficiency of large-scale dynamo action is determined by the product of $\alpha$ and $S$. However, as $S$ increases, $\alpha$ itself becomes quenched when $S$ becomes 
comparable with the inverse turnover time, i.e. $S \tau=O(1)$. Furthermore, the turbulent diffusivity becomes enhanced, suppressing the dynamo even further. Finally, it is found that the combined action of helicity and shear gives rise to a pumping velocity of mean magnetic field perpendicular to the plane of the shear flow. The existence of such a pumping velocity has not been emphasized before. On the other hand, it is well known that $\alpha \Omega$ (or rather $\alpha S$ ) dynamos can have travelling wave solutions (Brandenburg et al. 2001). When the product of $\alpha$ and $S$ is positive, these waves travel in the positive $z$ direction, which agrees with the direction of pumping. In the near-surface shear layer of the Sun this pumping would therefore support the equatorward migration in that layer.

It is important to understand the quenching of turbulent transport coefficients in the presence of shear beyond the kinematic approximation. In that case one needs to include the induction equation (in addition to the test field equations) and incorporate the resulting Lorentz force in the momentum equation (Brandenburg et al. 2008b). This would also help in alleviating problems of strong late-time fluctuations arising from the vorticity dynamo, because the vorticity dynamo tends to be suppressed by magnetic fields of equipartition strengths (Käpylä \& Brandenburg 2008). Similarly, given their potential importance in allowing the escape of magnetic helicity, the effects of open boundary conditions also needs to be considered. These questions are under study and will be reported elsewhere.

Acknowledgements. The authors acknowledge the hospitality of Nordita during the programme "Turbulence and Dynamos". A.B. and P.J.K. thank Astronomy Unit, Queen Mary University of London, for hospitality. Computational resources were granted by CSC (Espoo, Finland), UKMHD, and QMUL HPC facilities purchased under the SRIF initiative. This work was supported by the the Leverhulme Trust (DM, RT), the Academy of Finland grant No. 121431 (PJK), and the Swedish Research Council (AB).

\section{References}

Blackman, E., \& Field, G. B. 2000, ApJ, 534, 984

Blackman, E., \& Brandenburg, A. 2002, ApJ, 579, 359

Brandenburg, A. 2001, ApJ, 550, 824

Brandenburg, A. 2005, ApJ, 625, 539

Brandenburg, A. 2008, ApJ, submitted [arXiv:0808.0961]

Brandenburg, A., \& Sandin, C. 2004, A\&A, 427, 13

Brandenburg, A., \& Subramanian, K. 2005, Phys. Rep., 417, 1

Brandenburg, A., Bigazzi, A., \& Subramanian, K. 2001, MNRAS, 325, 685
Brandenburg, A., Rädler, K.-H., Rheinhardt, M., \& Käpylä, P. J. 2008a, ApJ, 676,740

Brandenburg, A., Rädler, K.-H., Rheinhardt, M., \& Subramanian, K. 2008b, ApJ, 687, L49

Brandenburg, A., Rädler, K.-H., \& Schrinner, M. 2008c, A\&A, 482, 739

Cattaneo, F., \& Hughes, D. W. 2008 [arXiv: 0805 . 2138]

Cattaneo, F., \& Tobias, S. M. 2008, J. Fluid Mech. [arXiv: 0809.1801]

Courvoisier, A. 2008, GAFD, 102, 217

Courvoisier, A., Hughes, D. W., \& Tobias, S. M. 2006, Phys. Rev. Lett., 96, 034503

Elperin, T., Kleeorin, N., \& Rogachevskii, I. 2003, Phys. Rev. E, 68, 016311

Field, G., \& Blackman, E. 2002, ApJ, 572, 685

Gruzinov, A. V., \& Diamond, P. H. 1994, Phys. Rev. Lett., 72, 1651

Haugen, N. E. L., Brandenburg, A., \& Dobler, W. 2004, Phys. Rev. E 70, 016308

Hubbard, A., \& Brandenburg, A. 2008 [arXiv: 0811.2561]

Hughes, D. W., \& Proctor, M. R. E. 2009, Phys. Rev. Lett. 102, 044501

Käpylä, P. J., \& Brandenburg, A. 2008, ApJ, submitted [arXiv: 0810. 2298]

Käpylä, P. J., Korpi, M. J., Ossendrijver, M., \& Stix, M. 2006, A\&A, 455, 401

Käpylä, P. J., Korpi, M. J., \& Brandenburg, A. 2008a, A\&A, 491, 353

Käpylä, P. J., Korpi, M. J., \& Brandenburg, A. 2008b, A\&A, submitted [arXiv: 0812.1792]

Käpylä, P. J., Mitra, D., \& Brandenburg, A. 2008, Phys. Rev. E, 79, 016302

Kitchatinov, L. L., Rüdiger, G., \& Pipin, V. V. 1994, AN, 315, 157

Kleeorin, N., Moss, D., Rogachevskii, I., \& Sokoloff, D. 2000, A\&A, 361, L5

Krause, F., \& Rädler, K.-H. 1980, Mean-field magnetohydrodynamics and dynamo theory (Oxford: Pergamon Press)

Leprovost, N., \& Kim, E.-J. 2008, Phys. Rev. Lett., 100, 144502

Ossendrijver, M., Stix, M., Brandenburg, A., \& Rüdiger, G. 2002, A\&A, 394, 735

Piddington, J. H. 1981 ApJ, 247, 291

Pipin, V. V., Rüdiger G., \& Kitchatinov, L. L. 1996, GAFD, 83, 119

Proctor, M. R. E. 2007, MNRAS, 382, L38

Rädler, K.-H., \& Stepanov, R. 2006, Phys. Rev. E, 73, 056311

Rädler, K.-H., \& Brandenburg, A. 2009, MNRAS, 393, 113

Rogachevskii, I., \& Kleeorin, N. 2003, Phys. Rev. E, 68, 036301

Rogachevskii, I., \& Kleeorin, N. 2004, Phys. Rev. E, 70, 046310

Rüdiger, G., \& Kitchatinov, L. 2006, Astron. Nachr., 327, 298

Schrinner, M., Rädler, K.-H., Schmitt, D., Rheinhardt, M., \& Christensen, U. 2005, Astron. Nachr., 326, 245

Schrinner, M., Rädler, K.-H., Schmitt, D., Rheinhardt, M., \& Christensen, U. 2007, GAFD, 101, 81

Subramanian, K., \& Brandenburg, A. 2004, Phys. Rev. Lett., 93, 205001

Sur, S., Brandenburg, A., \& Subramanian, K. 2008, MNRAS, 385, L15

Tilgner, A., \& Brandenburg, A. 2008, MNRAS, 391, 1477

Tobias, S. M., Brummell, N. H., Clune, T. L., \& Toomre, J. 1998, ApJ, 502, L177

Tobias, S. M., Brummell, N. H., Clune, T. L., \& Toomre, J. 2001, ApJ, 549, 1183

Vishniac, E. T., \& Brandenburg, A. 1997, ApJ, 584, L99

Vishniac, E. T., \& Cho, J. 2001, ApJ, 550, 752

Vainshtein, E. T., \& Cattaneo, J. 1992, ApJ, 393, 165

Yousef, T. A., Heinemann, T., Schekochihin, A. A., et al. 2008a, Phys. Rev. Lett., 100, 184501

Yousef, T. A., Heinemann, T., Rincon, F., et al. 2008b, AN, 329, 737 